\section{Patterns and predictors of aggressive incidents in children and adolescents admitted to a mental health in-patient unit}

\author{
A Dean', S Duke'2, M George ${ }^{2}$, J Scott ${ }^{2}$ \\ 'Kids in Mind Research, Mater Child \& Youth Mental Health Service; and \\ ${ }^{2}$ Mater Child \& Youth Mental Health Service Inpatient Unit, South Brisbane, Australia
}

Background: Aggression is a common clinical issue arising during treatment of young people with mental health problems. Poorly managed aggressive behaviours can compromise the therapeutic environment and pose a safety risk to patients and staff. The aim of this study was to understand patterns and predictors of aggressive behaviour in young people admitted to an in-patient psychiatric unit.

Methods: All patients admitted to the Mater Child \& Youth Mental Health Service Inpatient Unit between October 2004 and December 2005 were monitored for aggressive behaviours. Incidents were documented prospectively by clinical staff. Documentation incorporated nature of the incident, time, location and response. Demographic and clinical characteristics were collected from charts for all patients.

Results: During this time, 377 incidents were documented involving 40 patients (range 1-88 incidents per patient). About $23 \%$ of admissions were associated with aggressive behaviour. Thirty-six per cent of incidents involved violence toward staff. Compared with nonaggressive patients, aggressive patients were younger $(P<0.001)$, were more likely to be men $(P<0.01)$, have a history of aggression $(P<0.05)$ and were be more likely to be receiving psychotropic medications at admission $(P<0.001)$. Aggression patients were more likely to have a diagnosis of mental retardation, pervasive development disorders and attention deficit hyperactivity disorder (ADHD), and less likely to have a mood disorder $(P<0.001)$.

Conclusions: Young people most at risk of aggression appear to be younger men, with a diagnosis of mental retardation, development disorders or ADHD, and a history of aggressive behaviours. Further research is required to optimize prevention and management of these behaviours.

\section{Antidepressants in children and adolescents - changes in utilization after safety warnings}

\author{
A Dean', A Hendy², T McGuire ${ }^{2}$ \\ 'Kids in Mind Research, Mater Child \& Youth Mental Health Service; and \\ ${ }^{2}$ Mater Pharmacy Services, South Brisbane, Australia
}

Background: Antidepressants, in particular selective serotonin reuptake inhibitors (SSRIs), are one of the most commonly used classes of psychotropic drug in children and adolescents. In 2003, emerging evidence suggested that antidepressants may increase risk of suicidal behaviour in young people. This evidence was accompanied by national and international guidelines cautioning against use of many antidepressants in young people. This study aimed to assess whether these safety warnings have impacted upon antidepressant utilization rates.

Methods: This study was based at a metropolitan health service incorporating children's and adult hospitals. Total service utilization of antidepressants was extracted from pharmacy software for the period January 2002 to December 2005. Monthly utilization rates were computed for adults and children's services as defined daily doses per occupied bed days. Changes in utilization over time were compared for children and adults. Results: There was a significant relationship between time and antidepressant utilization in children and adolescents, where antidepressant use decreased over time $(R=0.416, t=-3.11, P<0.01)$. In contrast, there was a trend suggesting a minor increase in use of antidepressants over time in adults $(R=0.262$, $t=1.84, P=0.072$ ). In children, there was a reduction in use of all SSRIs, except for fluoxetine, which exhibited a small increase in use.

Conclusions: National and international warnings about safety of antidepressants in children and adolescent appear to have influenced utilization of these medications. Further research is required to determine optimal utilization rates.

\section{Orbitoprefrontal dysfunction and aggression in war veterans with post-traumatic stress disorder}

\section{J Dileo', M Creamer², V Anderson ${ }^{3}$, M Hopwood ${ }^{4}$, W Brewer ${ }^{5}$}

'Royal Children's Hospital; ${ }^{2}$ Australian Centre for Posttraumatic Mental Health; ${ }^{3}$ Murdoch Research Institute; ${ }^{4}$ Veterans Psychiatry Unit, Austin Health; and ${ }^{5}$ ORYGEN Research Centre, Melbourne, Australia

Objectives: To examine olfactory identification (OI) ability in war veterans with post-traumatic stress disorder (PTSD) as a probe of putative orbitoprefrontal dysfunction and to explore the utility of OI ability in predicting aggressive and impulsive behaviour in this clinical population.

Methods: Participants comprised 31 out-patient male war veterans with PTSD (mean $=58.23$ years, $\mathrm{SD}=2.56$ ) recruited from a Melbourne Veterans Psychiatry Unit, and 31 healthy age- and gender-matched controls $($ mean $=56.84$ years, $\mathrm{SD}=7.24)$. All 